PROCEEDINGS OF THE

AMERICAN MATHEMATICAL SOCIETY

Volume 135, Number 11, November 2007, Pages 3685-3693

S 0002-9939(07)08886-7

Article electronically published on June 22, 2007

\title{
ON THE STABILITY INDEX OF HYPERSURFACES WITH CONSTANT MEAN CURVATURE IN SPHERES
}

\author{
LUIS J. ALÍAS, ALDIR BRASIL, JR., AND OSCAR PERDOMO
}

(Communicated by Richard A. Wentworth)

\begin{abstract}
Barbosa, do Carmo and Eschenburg characterized the totally umbilical spheres as the only weakly stable compact constant mean curvature hypersurfaces in the Euclidean sphere $\mathbb{S}^{n+1}$. In this paper we prove that the weak index of any other compact constant mean curvature hypersurface $M^{n}$ in $\mathbb{S}^{n+1}$ which is not totally umbilical and has constant scalar curvature is greater than or equal to $n+2$, with equality if and only if $M$ is a constant mean curvature Clifford torus $\mathbb{S}^{k}(r) \times \mathbb{S}^{n-k}\left(\sqrt{1-r^{2}}\right)$ with radius $\sqrt{k /(n+2)} \leqslant r \leqslant \sqrt{(k+2) /(n+2)}$.
\end{abstract}

\section{INTRODUCTION}

Let $\psi: M^{n} \rightarrow \mathbb{S}^{n+1} \subset \mathbb{R}^{n+2}$ be a compact immersed orientable hypersurface of the unit Euclidean sphere $\mathbb{S}^{n+1}$. We will denote by $A$ its second fundamental form (with respect to a globally defined normal unit vector field $N$ ) and by $H$ its corresponding mean curvature, $H=(1 / n) \operatorname{tr}(A)$. Then, every smooth function $u \in \mathcal{C}^{\infty}(M)$ induces a normal variation $\psi_{t}$ of the immersion $\psi$, with variational normal field $u N$ and first variation of the area functional $A(t)$ given by

$$
\delta_{u} A=\left.\frac{d}{d t}\right|_{t=0} A(t)=-n \int_{M} u H \mathrm{~d} v,
$$

where $\mathrm{d} v$ stands for the area element of $M$. As a consequence, minimal hypersurfaces $(H=0)$ are characterized as critical points of the area functional whereas constant mean curvature hypersurfaces can be viewed as critical points of the area functional restricted to variations that preserve a certain volume function, that is, to smooth functions $u$ with mean value zero, $\int_{M} u \mathrm{~d} v=0$.

For such critical points, the stability equation of the corresponding variational problem is given by the second variation of the area functional,

$$
\delta_{u}^{2} A=\left.\frac{d^{2}}{d t^{2}}\right|_{t=0} A(t)=-\int_{M} u J(u) \mathrm{d} v,
$$

Received by the editors August 2, 2005 and, in revised form, August 11, 2006.

2000 Mathematics Subject Classification. Primary 53C42; Secondary 53A10.

Key words and phrases. Constant mean curvature, $H(r)$-torus, stability operator, first eigenvalue.

The first author was partially supported by MEC/FEDER project MTM2004-04934-C04-02, Spain, and by the Fundación Séneca project 00625/PI/04, Spain.

The second author was partially supported by CNPq, Brazil.

The third author was partially supported by Colciencias, Colombia. 
with $J(u)=\Delta u+\left(|A|^{2}+n\right) u$, where $\Delta$ stands for the Laplacian operator on $M$. The operator $J=\Delta+|A|^{2}+n$ is called the Jacobi or stability operator of the hypersurface (for the details see [2]).

The Jacobi operator induces the quadratic form $Q(u)=-\int_{M} u J(u) \mathrm{d} v$ acting on the space $\mathcal{C}^{\infty}(M)$ of smooth functions on $M$. In the case of minimal hypersurfaces, the index of a minimal hypersurface $M$, $\operatorname{Ind}(M)$, is defined as the maximum dimension of any subspace $V$ of $\mathcal{C}^{\infty}(M)$ on which $Q$ is negative definite. Equivalently, $\operatorname{Ind}(M)$ is the number of negative eigenvalues of $J$ (counted with multiplicity), which is necessarily finite and, intuitively, it measures the number of independent directions in which the hypersurface fails to minimize the area 11 If $\operatorname{Ind}(M)=0$, then $M$ is said to be stable.

Using $u \equiv 1$ as a test function, one observes that

$$
Q(1)=-\int_{M}\left(|A|^{2}+n\right) \mathrm{d} v=-n \operatorname{area}(M)-\int_{M}|A|^{2} \mathrm{~d} v \leqslant-n \operatorname{area}(M)<0 .
$$

In particular, $\operatorname{Ind}(M) \geqslant 1$ for every minimal hypersurface in $\mathbb{S}^{n+1}$, which means that there is no stable one. Even more, in $[8$ Simons proved that $\operatorname{Ind}(M)=1$ if and only if $M$ is a totally geodesic equator $\mathbb{S}^{n} \subset \mathbb{S}^{n+1}$.

It seems natural to ask oneself about the index of other minimal hypersurfaces in $\mathbb{S}^{n+1}$. Using the coordinates of the unit normal field $N$ as test functions, it is not difficult to see that if $M$ is not a totally geodesic equator, then $\operatorname{Ind}(M) \geqslant n+3$ (see [9] for $n=2$, and [5] and [7, Lemma 3.1] for general $n$ ). On the other hand, apart from the totally geodesic equators, the easiest minimal hypersurfaces in $\mathbb{S}^{n+1}$ are the minimal Clifford tori, and they all have $\operatorname{Ind}(M)=n+3$. For that reason it has been conjectured for a long time that minimal Clifford tori are the only minimal hypersurfaces in $\mathbb{S}^{n+1}$ with $\operatorname{Ind}(M)=n+3$. In 9 , Urbano showed that the conjecture is true when $n=2$. Later on, Guadalupe, Brasil Jr. and Delgado [6] showed that the conjecture is true for every dimension $n$, under the additional hypothesis of constant scalar curvature of $M$. More recently, Perdomo [7] proved that the conjecture is also true for every dimension $n$ with an additional assumption about the symmetries of $M$, and, in particular, the conjecture is true for minimal hypersurfaces with antipodal symmetry.

Our objective here is to consider the same kind of problems for the case of constant mean curvature hypersurfaces in $\mathbb{S}^{n+1}$. In [2, Barbosa, do Carmo and Eschenburg characterized the totally umbilical spheres $\mathbb{S}^{n}(r) \subset \mathbb{S}^{n+1}$ as the only weakly stable compact constant mean curvature hypersurfaces in $\mathbb{S}^{n+1}$ (see the next section for the precise meaning of weak stability and weak index). Then, our main result (Theorem 11) establishes that the weak index of any other compact constant mean curvature hypersurface $M^{n}$ in $\mathbb{S}^{n+1}$ which is not totally umbilical and has constant scalar curvature is greater than or equal to $n+2$, with equality if and only if $M$ is a constant mean curvature Clifford torus $\mathbb{S}^{k}(r) \times \mathbb{S}^{n-k}\left(\sqrt{1-r^{2}}\right)$ with radius $\sqrt{k /(n+2)} \leqslant r \leqslant \sqrt{(k+2) /(n+2)}$.

\section{Stability AND INDEX OF CONSTANT MEAN CURVATURE HyPERSURFACES}

In contrast to the case of minimal hypersurfaces, in the case of hypersurfaces with constant mean curvature one can consider two different eigenvalue problems:

\footnotetext{
${ }^{1}$ Observe that with our criterion, a real number $\lambda$ is an eigenvalue of $J$ if and only if $J u+\lambda u=0$ for some smooth function $u \in \mathcal{C}^{\infty}(M), u \not \equiv 0$.
} 
the usual Dirichlet problem, associated with the quadratic form $Q$ acting on the whole space of smooth functions on $M$, and the so-called twisted Dirichlet problem, associated with the same quadratic form $Q$, but restricted to the subspace of smooth functions $u \in \mathcal{C}^{\infty}(M)$ satisfying the additional condition $\int_{M} u \mathrm{~d} v=0$.

Similarly, there are two different notions of stability and index, the strong stability and strong index, denoted by $\operatorname{Ind}(M)$ and associated to the usual Dirichlet problem, and the weak stability and weak index, denoted by $\operatorname{Ind}_{T}(M)$ and associated to the twisted Dirichlet problem. Specifically, the strong index is simply the maximum dimension of any subspace $V$ of $\mathcal{C}^{\infty}(M)$ on which $Q$ is negative definite, and $M$ is called strongly stable if and only if $\operatorname{Ind}(M)=0$. On the other hand, the weak index is the maximum dimension of any subspace $V$ of $\mathcal{C}_{T}^{\infty}(M)$ on which $Q$ is negative definite, where $\mathcal{C}_{T}^{\infty}(M)=\left\{u \in \mathcal{C}^{\infty}(M): \int_{M} u \mathrm{~d} v=0\right\}$, and $M$ is called weakly stable if and only if $\operatorname{Ind}_{T}(M)=0$. As observed by Barbosa and Bérard [1], both eigenvalue problems are interesting and they have similar and related properties. We refer the reader to [1] for a detailed study of the relationship between these two eigenvalue problems and their corresponding stability and index notions.

From a geometrical point of view, the weak index is more natural than the strong index. However, from an analytical point of view, the strong index is more natural and easier to use. For instance, observe that for a hypersurface with constant mean curvature $H$ in $\mathbb{S}^{n+1}$ the Jacobi operator can be written as $J=\Delta+|\phi|^{2}+n\left(1+H^{2}\right)$, where $|\phi|^{2}=|A|^{2}-n H^{2} \geqslant 0$, and $|\phi|^{2} \equiv 0$ if and only of $M$ is totally umbilical 2 Therefore, using again $u \equiv 1$ as a test function for estimating $\operatorname{Ind}(M)$, one observes that

$$
\begin{aligned}
Q(1) & =-\int_{M}\left(|\phi|^{2}+n\left(1+H^{2}\right)\right) \mathrm{d} v=-n\left(1+H^{2}\right) \operatorname{area}(M)-\int_{M}|\phi|^{2} \mathrm{~d} v \\
& \leqslant-n\left(1+H^{2}\right) \operatorname{area}(M)<0 .
\end{aligned}
$$

In particular, $\operatorname{Ind}(M) \geqslant 1$ for every constant mean curvature hypersurface in $\mathbb{S}^{n+1}$, which means that no constant mean curvature hypersurface is strongly stable.

\section{Constant mean CURVATURe hypersurfaces With LOW INDEX IN $\mathbb{S}^{n+1}$}

In [2] Barbosa, do Carmo and Eschenburg characterized the totally umbilical spheres $\mathbb{S}^{n}(r) \subset \mathbb{S}^{n+1}$ as the only weakly stable compact hypersurfaces with constant mean curvature in $\mathbb{S}^{n+1}$. Equivalently, $\operatorname{Ind}_{T}(M)=0$ for the totally umbilical spheres in $\mathbb{S}^{n+1}$, and $\operatorname{Ind}_{T}(M) \geqslant 1$ for the rest of the constant mean curvature compact hypersurfaces of $\mathbb{S}^{n+1}$.

Apart from the totally umbilical spheres, the easiest constant mean curvature hypersurfaces in $\mathbb{S}^{n+1}$ are the constant mean curvature Clifford tori. A constant mean curvature Clifford torus in $\mathbb{S}^{n+1}$ is obtained by considering the standard immersions $\mathbb{S}^{k}(r) \hookrightarrow \mathbb{R}^{k+1}$ and $\mathbb{S}^{n-k}\left(\sqrt{1-r^{2}}\right) \hookrightarrow \mathbb{R}^{n-k+1}$, for a given radius $0<$ $r<1$ and integer $k \in\{1, \ldots, n-1\}$, and taking the product immersion $\mathbb{S}^{k}(r) \times$ $\mathbb{S}^{n-k}\left(\sqrt{1-r^{2}}\right) \hookrightarrow \mathbb{S}^{n+1} \subset \mathbb{R}^{n+2}$. Its principal curvatures are given by

$$
\kappa_{1}=\cdots=\kappa_{k}=-\frac{\sqrt{1-r^{2}}}{r}, \quad \kappa_{k+1}=\cdots=\kappa_{n}=\frac{r}{\sqrt{1-r^{2}}}
$$

\footnotetext{
${ }^{2}$ This property about $|\phi|^{2}$ will be used later on several times.
} 
and its constant mean curvature $H=H(r)$ is given by

$$
n H(r)=\frac{n r^{2}-k}{r \sqrt{1-r^{2}}}
$$

In particular, $H(r)=0$ precisely when $r=\sqrt{k / n}$, which corresponds to the minimal Clifford tori above.

For the constant mean curvature Clifford tori, one gets

$$
|A|^{2}+n=\frac{k}{r^{2}}+\frac{n-k}{1-r^{2}}
$$

and the Jacobi operator reduces to

$$
J=\Delta+\left(\frac{k}{r^{2}}+\frac{n-k}{1-r^{2}}\right) .
$$

In particular, the spectrum of $J$ is directly related to the spectrum of $\Delta$; specifically, they share the same eigenfunctions, and their eigenvalues are related by

$$
\lambda_{i}^{J}=\lambda_{i}^{\Delta}-\left(\frac{k}{r^{2}}+\frac{n-k}{1-r^{2}}\right), \quad i=1,2, \ldots
$$

As a consequence, using that $\lambda_{1}^{\Delta}=0$ and its corresponding eigenfunctions are the constant functions, we know that $\lambda_{1}^{J}=-\left(\frac{k}{r^{2}}+\frac{n-k}{1-r^{2}}\right)$ with multiplicity 1 and its corresponding eigenfunctions are the constant functions. Observe that $\lambda_{1}^{J}=-\left(\frac{k}{r^{2}}+\frac{n-k}{1-r^{2}}\right)<0$ contributes to $\operatorname{Ind}(M)$ but not to $\operatorname{Ind}_{T}(M)$, because its eigenfunctions do not satisfy the restriction $\int_{M} u \mathrm{~d} v=0$. Even more, since all the rest of the eigenfunctions of $J$ are orthogonal to the constant functions, they do satisfy the restriction $\int_{M} u \mathrm{~d} v=0$ and do contribute to $\operatorname{Ind}_{T}(M)$. Therefore, in this case we have $\operatorname{Ind}(M)=\operatorname{Ind}_{T}(M)+1$, and $\operatorname{Ind}_{T}(M)$ reduces to the number of positive eigenvalues of the Laplacian operator (counted with multiplicity) that are strictly less than $\frac{k}{r^{2}}+\frac{n-k}{1-r^{2}}$.

To compute it, we simply need to recall the fact that if $\lambda$ is an eigenvalue of the Laplacian on $\mathbb{S}^{k}(r)$ with multiplicity $m_{\lambda}$ and $\mu$ is an eigenvalue of the Laplacian on $\mathbb{S}^{n-k}\left(\sqrt{1-r^{2}}\right)$ with multiplicity $m_{\mu}$, then $\nu=\lambda+\mu$ is an eigenvalue of the Laplacian on $M=\mathbb{S}^{k}(r) \times \mathbb{S}^{n-k}\left(\sqrt{1-r^{2}}\right)$, and the multiplicity of $\nu$ is the sum of the products $m_{\lambda} m_{\mu}$ for all the possible values of $\lambda$ and $\mu$ satisfying $\nu=\lambda+\mu[3$. In our case, the eigenvalues of the Laplacian on $\mathbb{S}^{k}(r)$ are

$$
\lambda_{i}=\frac{(i-1)(k+i-2)}{r^{2}}, \quad i=1,2,3, \ldots,
$$

with multiplicities

$$
m_{\lambda_{1}}=1, \quad, m_{\lambda_{2}}=k+1,
$$

and

$$
m_{\lambda_{i}}=\left(\begin{array}{c}
k+i-1 \\
i-1
\end{array}\right)-\left(\begin{array}{c}
k+i-3 \\
i-3
\end{array}\right), \quad i=3,4, \ldots,
$$

and the eigenvalues of the Laplacian on $\mathbb{S}^{n-k}\left(\sqrt{1-r^{2}}\right)$ are

$$
\mu_{j}=\frac{(j-1)(n-k+j-2)}{1-r^{2}}, \quad j=1,2,3, \ldots,
$$

with multiplicities

$$
m_{\mu_{1}}=1, \quad, m_{\mu_{2}}=n-k+1
$$


and

$$
m_{\mu_{j}}=\left(\begin{array}{c}
n-k+j-1 \\
j-1
\end{array}\right)-\left(\begin{array}{c}
n-k+j-3 \\
j-3
\end{array}\right), \quad j=3,4, \ldots
$$

and the problem reduces to counting when $\lambda_{i}+\mu_{j}<\frac{k}{r^{2}}+\frac{n-k}{1-r^{2}}=\lambda_{2}+\mu_{2}$, with $i=1, j>1$, and $i>1, j=1$.

Observe that we always have $\lambda_{1}+\mu_{2}=\frac{n-k}{1-r^{2}}<\frac{k}{r^{2}}+\frac{n-k}{1-r^{2}}$ with multiplicity $n-k+1$ and $\lambda_{2}+\mu_{1}=\frac{k}{r^{2}}<\frac{k}{r^{2}}+\frac{n-k}{1-r^{2}}$ with multiplicity $k+1$. Therefore, $\operatorname{Ind}_{T}(M) \geqslant n+2$ for every constant mean curvature Clifford torus. Moreover, $\operatorname{Ind}_{T}(M)=n+2$ precisely when

$$
\lambda_{1}+\mu_{3}=\mu_{3} \geqslant \frac{k}{r^{2}}+\frac{n-k}{1-r^{2}} \quad \text { and } \quad \lambda_{3}+\mu_{1}=\lambda_{3} \geqslant \frac{k}{r^{2}}+\frac{n-k}{1-r^{2}},
$$

that is, if and only if

$$
\frac{k}{n+2} \leqslant r^{2} \leqslant \frac{k+2}{n+2} .
$$

Observe that, in particular, this happens when $r^{2}=k / n$, so that the minimal Clifford tori satisfy $\operatorname{Ind}_{T}(M)=n+2$ when regarded as constant mean curvature hypersurfaces.

Motivated by the value of $\operatorname{Ind}_{T}(M)$ for the constant mean curvature Clifford tori, in this paper we will prove the following result.

Theorem 1. Let $M$ be a compact orientable hypersurface immersed in the Euclidean sphere $\mathbb{S}^{n+1}$ with constant mean curvature $H$, and let $\operatorname{Ind}_{T}(M)$ stand for its weak stability index. Assume that $M$ has constant scalar curvature. Then

(i) either $\operatorname{Ind}_{T}(M)=0$ (and $M$ is a totally umbilic sphere in $\mathbb{S}^{n+1}$ ), or

(ii) $\operatorname{Ind}_{T}(M) \geqslant n+2$, with equality if and only if $M$ is a constant mean curvature Clifford torus $\mathbb{S}^{k}(r) \times \mathbb{S}^{n-k}\left(\sqrt{1-r^{2}}\right)$ with radius $\sqrt{k /(n+2)} \leqslant r \leqslant$ $\sqrt{(k+2) /(n+2)}$.

It is worth pointing out that the value of the index of the constant mean curvature Clifford tori $\mathbb{S}^{k}(r) \times \mathbb{S}^{n-k}\left(\sqrt{1-r^{2}}\right)$ converges to infinity as $r$ converges either to 0 or 1. For that reason, it is not possible, in general, to find a characterization of all constant mean curvature Clifford tori in terms of their index. Actually, it can be easily seen that for every $i, j \geqslant 3$,

$$
\lambda_{1}+\mu_{j}<\frac{k}{r^{2}}+\frac{n-k}{1-r^{2}}
$$

if and only if

$$
r^{2}<a_{j}=\frac{k}{n+(j-3)(n-k)+(j-1)(j-2)},
$$

and

if and only if

$$
\lambda_{i}+\mu_{1}<\frac{k}{r^{2}}+\frac{n-k}{1-r^{2}}
$$

$$
r^{2}>b_{i}=1-\frac{(n-k)}{(n-k)+(i-2)(k+i-1)},
$$

where $\left\{a_{j}\right\} \searrow 0$ and $\left\{b_{i}\right\} \nearrow 1$. As a consequence, if $\operatorname{Ind}_{T}(r)$ stands for the weak stability index of $\mathbb{S}^{k}(r) \times \mathbb{S}^{n-k}\left(\sqrt{1-r^{2}}\right)$, we obtain that $\operatorname{Ind}_{T}(r)=n+2$ when 


$$
\begin{aligned}
a_{3}= & k /(n+2) \leqslant r^{2} \leqslant(k+2) /(n+2)=b_{3}, \\
& \operatorname{Ind}_{T}(r)=n+2+\sum_{l=3}^{j} m_{\mu_{l}}=k+\left(\begin{array}{c}
n-k+j-1 \\
j-1
\end{array}\right)+\left(\begin{array}{c}
n-k+j-2 \\
j-2
\end{array}\right)
\end{aligned}
$$

when $a_{j+1} \leqslant r^{2}<a_{j}$, with $j \geqslant 3$, and

$$
\operatorname{Ind}_{T}(r)=n+2+\sum_{l=3}^{i} m_{\lambda_{l}}=n-k+1+\left(\begin{array}{c}
k+i-1 \\
i-1
\end{array}\right)+\left(\begin{array}{c}
k+i-2 \\
i-2
\end{array}\right)
$$

when $b_{i}<r^{2} \leqslant b_{i+1}$, with $i \geqslant 3$.

\section{Proof of Theorem 1}

We already know from 2 that $\operatorname{Ind}_{T}(M)=0$ for the totally umbilical spheres in $\mathbb{S}^{n+1}$, and $\operatorname{Ind}_{T}(M) \geqslant 1$ for the rest of the constant mean curvature hypersurfaces of $\mathbb{S}^{n+1}$. Our first objective now is to prove that, if the scalar curvature of $M$ is constant (or equivalently, $|A|^{2}$ is constant), then $\operatorname{Ind}_{T}(M) \geqslant n+2$ for any constant mean curvature hypersurface of $\mathbb{S}^{n+1}$ that is not totally umbilical. To see this, we need to find a subspace $V$ of $\mathcal{C}_{T}^{\infty}(M)=\left\{u \in \mathcal{C}^{\infty}(M): \int_{M} u=0\right\}$ with $\operatorname{dim} V \geqslant n+2$ on which $Q$ is negative definite.

For a fixed arbitrary vector $v \in \mathbb{R}^{n+2}$, we will work with the functions $\ell_{v}=\langle\psi, v\rangle$ and $f_{v}=\langle N, v\rangle$ defined on $M$. Observe that their gradients are given by

$$
\nabla \ell_{v}=v^{\top} \text { and } \nabla f_{v}=-A\left(v^{\top}\right),
$$

where $v^{\top} \in \mathcal{X}(M)$ denotes the tangent component of $v$ along the immersion $\psi$,

$$
v=v^{\top}+f_{v} N+\ell_{v} \psi
$$

Therefore, for every $X \in \mathcal{X}(M)$ we have

$$
\nabla_{X} \nabla \ell_{v}=\nabla_{X} v^{\top}=-\ell_{v} X+f_{v} A(X),
$$

so that

$$
\Delta \ell_{v}=\operatorname{tr}\left(\nabla^{2} \ell_{v}\right)=-n \ell_{v}+n H f_{v}
$$

and

$$
J \ell_{v}=|A|^{2} \ell_{v}+n H f_{v} .
$$

On the other hand, using the Codazzi equation,

$$
\begin{aligned}
\nabla_{X} \nabla f_{v} & =-\nabla_{X}\left(A\left(v^{\top}\right)\right)=-\left(\nabla_{X} A\right)\left(v^{\top}\right)-A\left(\nabla_{X} v^{\top}\right) \\
& =-\left(\nabla_{v^{\top}} A\right)(X)+\ell_{v} A(X)-f_{v} A^{2}(X) .
\end{aligned}
$$

Therefore

$$
\begin{aligned}
\Delta f_{v} & =-\operatorname{tr}\left(\nabla_{v^{\top}} A\right)+n H \ell_{v}-|A|^{2} f_{v}=-n\left\langle v^{\top}, \nabla H\right\rangle+n H \ell_{v}-|A|^{2} f_{v} \\
& =n H \ell_{v}-|A|^{2} f_{v},
\end{aligned}
$$

since the mean curvature $H$ is constant, and

$$
J f_{v}=n H \ell_{v}+n f_{v} .
$$

When $H=0$, then we take

$$
V=\left\{f_{v}: v \in \mathbb{R}^{n+2}\right\} .
$$


In fact, it follows from (5) that the functions $f_{v}$ are eigenfunctions of $J$ with negative eigenvalue $-n$ and, since $|A|^{2}>0$ is constant, they also satisfy the condition $\int_{M} f_{v}=0$ by (44). We also claim that $\operatorname{dim} V \geqslant n+2$. Otherwise there would exist a unit vector $v \in \mathbb{R}^{n+2}$ such that $f_{v}=0$, which would imply that $M$ is necessarily a totally geodesic equator of $\mathbb{S}^{n+1}$ (see the proof of [7, Lemma 3.1]).

Therefore, assume that $H \neq 0$ and consider test functions of the form $\ell_{v}-$ $\alpha f_{v}$, where $\alpha \in \mathbb{R}$ is a real constant. We will force the functions $\ell_{v}-\alpha f_{v}$ to be eigenfunctions of $J$. From (3) and (5) we see that

$$
J\left(\ell_{v}-\alpha f_{v}\right)=\left(|A|^{2}-n H \alpha\right) \ell_{v}+n(H-\alpha) f_{v} .
$$

Thus, if $\alpha$ is a solution of the quadratic equation

$$
n H \alpha^{2}+\left(n-|A|^{2}\right) \alpha-n H=0,
$$

then we can easily see that $\ell_{v}-\alpha f_{v}$ is an eigenfunction of $J$ with eigenvalue $\lambda=$ $n H \alpha-|A|^{2}$. Equation (6) has two different real roots,

$$
\alpha_{ \pm}=\frac{|A|^{2}-n \pm \sqrt{D}}{2 n H}, \quad \text { where } \quad D=\left(|A|^{2}-n\right)^{2}+4 n^{2} H^{2}>0
$$

with corresponding values of $\lambda$ given by

$$
\lambda_{ \pm}=n H \alpha_{ \pm}-|A|^{2}=\frac{-\left(n+|A|^{2}\right) \pm \sqrt{D}}{2} .
$$

Observe that

$$
\lambda_{-}=\frac{-\left(n+|A|^{2}\right)-\sqrt{D}}{2}<\lambda_{+}=\frac{-\left(n+|A|^{2}\right)+\sqrt{D}}{2}<0
$$

because of $|A|^{2}-n H^{2}>0$. Therefore, $\lambda_{-}$and $\lambda_{+}$are negative eigenvalues of $J$. Set

$$
U_{ \pm}=\left\{\ell_{v}-\alpha_{ \pm} f_{v}: v \in \mathbb{R}^{n+2}\right\}
$$

Then, $J u+\lambda_{ \pm} u=0$ for every $u \in U_{ \pm}$. Moreover, functions belonging to $U_{ \pm}$also satisfy the condition $\int_{M} f=0$. To see this, observe that if $u \in U_{ \pm}$, then

$$
\Delta u+\mu_{ \pm} u=0
$$

with $\mu_{ \pm}=n+|A|^{2}+\lambda_{ \pm}$, that is,

$$
\mu_{+}=-\lambda_{-}>-\lambda_{+}=\mu_{-}>0 .
$$

That is, functions belonging to $U_{ \pm}$are also nonconstant eigenfunctions of the Laplacian and then satisfy the condition $\int_{M} u=0$. It follows from here that

$$
\operatorname{Ind}_{T}(M) \geqslant \operatorname{dim}\left(U_{-} \oplus U_{+}\right)=\operatorname{dim} U_{-}+\operatorname{dim} U_{+},
$$

where the last equality comes from the fact that $U_{-}$and $U_{+}$are in fact $L^{2}$ orthogonal subspaces because they are eigenspaces of the Laplacian associated to different eigenvalues. It remains to estimate $\operatorname{dim} U_{-}$and $\operatorname{dim} U_{+}$. To do that, consider the linear epimorphisms $\varphi_{ \pm}: \mathbb{R}^{n+2} \rightarrow U_{ \pm}$given by

$$
\varphi_{ \pm}(v)=\ell_{v}-\alpha_{ \pm} f_{v}
$$

Clearly,

$$
\operatorname{dim} U_{ \pm}=n+2-\operatorname{dim} \operatorname{ker} \varphi_{ \pm} .
$$

We claim that $\operatorname{ker} \varphi_{-} \cap \operatorname{ker} \varphi_{+}=\{\mathbf{0}\}$. To see this, assume that there exists a unit vector $v \in \operatorname{ker} \varphi_{-} \cap \operatorname{ker} \varphi_{+}$. Then

$$
\ell_{v}-\alpha_{-} f_{v}=0=\ell_{v}-\alpha_{+} f_{v}
$$


implies that

$$
\alpha_{+}\left(\ell_{v}-\alpha_{-} f_{v}\right)=0=\alpha_{-}\left(\ell_{v}-\alpha_{+} f_{v}\right),
$$

that is, $\left(\alpha_{+}-\alpha_{-}\right) \ell_{v}=0$ and equivalently $\ell_{v}=0$, since $\alpha_{+}-\alpha_{-}>0$. But this would mean that $M$ is a totally geodesic equator of $\mathbb{S}^{n+1}$, which is not possible. Summing up, $\operatorname{ker} \varphi_{-} \cap \operatorname{ker} \varphi_{+}=\{\mathbf{0}\}$ and then

$$
\operatorname{dim} \operatorname{ker} \varphi_{-}+\operatorname{dim} \operatorname{ker} \varphi_{+}=\operatorname{dim}\left(\operatorname{ker} \varphi_{-} \oplus \operatorname{ker} \varphi_{+}\right) \leqslant n+2,
$$

which gives

$$
\operatorname{Ind}_{T}(M) \geqslant \operatorname{dim} U_{-}+\operatorname{dim} U_{+}=2(n+2)-\left(\operatorname{dim} \operatorname{ker} \varphi_{-}+\operatorname{dim} \operatorname{ker} \varphi_{+}\right) \geqslant n+2 .
$$

This finishes the proof of the inequality in (ii) of Theorem 1 .

Assume now that equality holds, $\operatorname{Ind}_{T}(M)=n+2$. Then $\operatorname{dim}\left(\operatorname{ker} \varphi_{-} \oplus \operatorname{ker} \varphi_{+}\right)=$ $n+2$, which means that $\mathbb{R}^{n+2}$ splits as a direct sum of its subspaces $\operatorname{ker} \varphi_{-}$and $\operatorname{ker} \varphi_{+}, \mathbb{R}^{n+2}=\operatorname{ker} \varphi_{-} \oplus \operatorname{ker} \varphi_{+}$. Then, at any point $p \in M$ we have that the tangent space $T_{p} M$ splits also as a direct sum of two subspaces

$$
T_{p} M=T_{p} M \cap \mathbb{R}^{n+2}=T_{p} M^{-} \oplus T_{p} M^{+},
$$

where $T_{p} M^{-}=T_{p} M \cap \operatorname{ker} \varphi_{-}$and $T_{p} M^{+}=T_{p} M \cap \operatorname{ker} \varphi_{+}$. Assume that $T_{p} M^{-} \neq$ $\{\mathbf{0}\}$ and let $v \in T_{p} M^{-}, v \neq 0$. Then $\ell_{v}-\alpha_{-} f_{v}=0$ on $M$, which implies that necessarily $\alpha_{-} \neq 0$. Otherwise, $\ell_{v}=0$ and $M$ would be a totally geodesic equator, which is not possible because we are assuming $\operatorname{Ind}_{T}(M)=n+2$. Moreover, from (11) we also have that

$$
\nabla\left(\ell_{v}-\alpha_{-} f_{v}\right)=v^{\top}+\alpha_{-} A\left(v^{\top}\right)=0 \quad \text { on } \quad M .
$$

In particular, at the point $p$ we have that $v^{\top}(p)=v$ and

$$
A_{p}(v)=-\frac{1}{\alpha_{-}} v
$$

Summing up, if $T_{p} M^{-} \neq\{\mathbf{0}\}$, then $\alpha_{-} \neq 0$ and $T_{p} M^{-}$is a subspace of $T_{p} M$ of principal directions with constant principal curvature $-1 / \alpha_{-}$. Similarly, if $T_{p} M^{+} \neq$ $\{\mathbf{0}\}$, then $\alpha_{+} \neq 0$ and $T_{p} M^{+}$is a subspace of $T_{p} M$ of principal directions with constant principal curvature $-1 / \alpha_{+}$.

Notice that if for some point $p_{0} \in M$ it follows that either $T_{p_{0}} M^{+}=\{\mathbf{0}\}$ or $T_{p_{0}} M^{-}=\{\mathbf{0}\}$, then either $T_{p_{0}} M=T_{p_{0}} M^{-}$or $T_{p_{0}} M=T_{p_{0}} M^{+}$, respectively, and $p_{0}$ would be an umbilical point, that is, $|\phi|^{2}\left(p_{0}\right)=0$. But $|\phi|^{2}$ being constant on $M$ this implies that $M$ would be totally umbilical, which is not possible because we are assuming $\operatorname{Ind}_{T}(M)=n+2$. Therefore, $M$ has two constant principal curvatures $-1 / \alpha_{-}$and $-1 / \alpha_{+}$at any point. As a consequence, $M$ is a compact hypersurface of $\mathbb{S}^{n+1}$ with two different and nonzero constant principal curvatures, and by a well-known rigidity result by Cartan (see, for instance, Chapter 3 in [4]) we conclude that $M$ is a constant mean curvature Clifford torus of the form $\mathbb{S}^{k}(r) \times$ $\mathbb{S}^{n-k}\left(\sqrt{1-r^{2}}\right)$ with radius $0<r<1$. Finally, from our previous discussion about the values of $\operatorname{Ind}_{T}(M)$ in Section 2, we conclude that it must be $\sqrt{k /(n+2)} \leqslant r \leqslant$ $\sqrt{(k+2) /(n+2)}$. 


\section{ACKNOWLEDGMENTS}

This work was started while the first and third authors were visiting the Departamento de Matemática of the Universidade Federal do Ceará in Fortaleza, Brasil. They would like to thank that institution and the members of the department for their hospitality.

\section{REFERENCES}

[1] J.L. Barbosa and P. Bérard, Eigenvalue and "twisted" eigenvalue problems. Applications to CMC surfaces, J. Math. Pures Appl. (9) 79 (2000), 427-450. MR1759435 (2001f:58064)

[2] J.L. Barbosa, M. do Carmo and J. Eschenburg, Stability of hypersurfaces with constant mean curvature in Riemannian manifolds, Math. Z. 197 (1988), 123-138. MR917854 (88m:53109)

[3] M. Berger, P. Gauduchon, and E. Mazet, Le spectre d'une variété riemannienne. Lecture Notes in Mathematics, Vol. 194, Springer-Verlag, Berlin-New York, 1971. MR0282313 $(43: 8025)$

[4] T.E. Cecil and P.J. Ryan, Tight and taut immersions of manifolds. Research Notes in Mathematics, 107. Pitman (Advanced Publishing Program), Boston, MA, 1985. MR781126 (87b:53089)

[5] A. El Soufi, Applications harmoniques, immersions minimales et transformations conformes de la sphère, Compositio Math. 85 (1993), 281-298. MR1214448 (94h:58058)

[6] I. Guadalupe, A. Brasil, Jr., and J.A. Delgado, A characterization of the Clifford torus. Rend. Circ. Mat. Palermo (2) 48 (1999), 537-540. MR1731453 (2001f:53111)

[7] O. Perdomo, Low index minimal hypersurfaces of spheres, Asian J. Math. 5 (2001), 741-749. MR1913819 (2003e:53080)

[8] J. Simons, Minimal varieties in Riemannian manifolds, Ann. of Math. (2), 88 (1968) 62-105. MR0233295 (38:1617)

[9] F. Urbano, Minimal surfaces with low index in the three-dimensional sphere. Proc. Amer. Math. Soc. 108 (1990), 989-992. MR1007516 (90h:53073)

Departamento de Matemáticas, Universidad de Murcia, Campus de Espinardo, E-30100 Espinardo, Murcia, Spain

E-mail address: ljalias@um.es

Departamento de Matemática, Universidade Federal do Ceará, Campus do Pici, 60455-760 Fortaleza-Ce, Brazil

E-mail address: aldir@mat.ufc.br

Departamento de Matemáticas, Universidad del Valle, Cali, Colombia

E-mail address: osperdom@mafalda.univalle.edu.co 